\title{
Trends in occupational injuries and diseases among Saudi and non-Saudi insured workers
}

\author{
Mohsin Abbas, ${ }^{1}$ Muhammad Kashif, ${ }^{2}$ Mansour Balkhyour, ${ }^{3}$ Ijaz Ahmad, ${ }^{3,4}$ Zaki-ul-Zaman Asam ${ }^{1}$ and Rashid Saeed ${ }^{1}$
}

${ }^{1}$ Department of Environmental Sciences, Faculty of Sciences, University of Gujrat, Hafiz Hayat Campus, Jalalpur Road, Gujrat, Pakistan (Correspondence to: M.Abbas: mohsinabbas34@yahoo.com; mohsin.abbas@uog.edu.pk). ${ }^{2}$ Department of Mathematics and Statistics, University of Agriculture Faisalabad, Faisalabad, Pakistan. ${ }^{3}$ Department of Environmental Sciences, Faculty of Meteorology Environment Arid Land Agriculture, King Abdul-Aziz University, Jeddah, Saudi Arabia. ${ }^{4}$ Center of Excellence in Environmental Studies, King Abdul-Aaziz University, Jeddah, Saudi Arabia.

\begin{abstract}
Background: The ongoing industrial and infrastructural development in Saudi Arabia carries a high risk of occupational injuries/diseases.

Aims: To compare trends in occupational injuries and diseases among Saudi and non-Saudi insured workers.

Methods: We used the index values method and slope (S) calculation to analyse the occupational injuries/diseases trends from data in the annual statistical reports published by the General Organization for Social Insurance between 2004 and 2014. Data for 10565993 (18.5\%) Saudi insured workers (SIWs) and 46402079 (81.5\%) non-Saudi insured workers (NSIWs) and 896627 occupational injuries/diseases were analysed.

Results: The distribution of incidences of occupational injuries/diseases among NSIWs (93.5\%) was 14 times higher than that of SIWs (6.5\%). Occupational injuries/diseases were more likely to increase among NSIWs than SIWs, particularly in the construction, trade, financing and real estate economic activities, and engineering, technicians and service workers occupations. There was a general decreasing trend in occupational injuries/diseases until 2013.
\end{abstract}

Conclusions: Non-Saudi insured workers were at high risk of occupational injuries/diseases, therefore, implementation of an effective injury prevention programme is required.

Keywords: occupational injuries, occupational diseases, insured workers, Saudi Arabia, migrant workers

Citation: Abbas M; Kashif M; Balkhyour M; Ahmad I; Asam Z; Saeed R. Trends in occupational injuries and diseases among Saudi and non-Saudi insured workers. East Mediterr Health J. 2018;24(10):1010-1017. https://doi.org/10.26719/2018.24.10.1010

Received: 18/07/17; accepted: 21/11/17

Copyright (C) World Health Organization (WHO) 2018. Some rights reserved. This work is available under the CC BY-NC-SA 3.o IGO license (https:// creativecommons.org/licenses/by-nc-sa/3.o/igo).

\section{Introduction}

The incidence of occupational injuries among migrant workers is reported as high as compared to that of the native working population (1-5). In a recent review of migrant workers of 16 countries, the percentage of occupational injuries was twice that of native workers (6). Migrant workers are also less aware of workplace safety and have little knowledge about their occupational rights (injury compensation or other financial benefits) (7). Saudi Arabia has > 9 million migrant workers according to a recent Labor Force Survey (2016_Q2) (8). The Population Division of the United Nations ranked Saudi Arabia in fourth position regarding number of migrant workers. Previously, a 4 times higher incidence of occupational injuries/diseases was found among migrant workers as compared to native Saudi workers in Saudi Arabia.

The present study analysed and compared the trends in occupational injuries/diseases between non-Saudi insured workers (NSIWs) and Saudi insured workers (SIWs) between 2004 and 2014 in Saudi Arabia.

\section{Methods}

We used an open data source in the form of published annual statistical reports between 2004 and 2014 available on the website of General Organization for Social
Insurance (GOSI) (http://www.gosi.gov.sa/). The original data for occupational injuries from Arabic statistical reports were translated into English and transformed into MS Excel format for analysis. The index value calculation method was used to determine the trends in occupational injuries/diseases in NSIWs and SIWs between 2004 and 2014. The index values calculation method had 2 different types of implications: (1) the occupational injuries/diseases trends based on the reference year 2004; and (2) occupational injuries/diseases trends for NSIWs based on the occupational injuries/diseases of SIWs for each variable. Index value yearly trends in occupational injuries/diseases of both SIWs and NSIWs were calculated for each type of economic activity and major occupation in 2004 and 2014 by considering the respective occupational injuries/diseases value of the year 2004, and calculation of the slope value $(S)$ displayed the upward and downward trends in occupational injuries/diseases. $S$ was used to describe both direction and steepness of the data (9). A negative value of $S$ showed a decline in occupational injuries/ diseases, and a positive value of $S$ showed an increase. According to GOSI, occupational (employment) injuries are: (1) any accident incurred by the worker during performance or because of work; (2) any accident incurred by the worker on their way from their dwelling to their workplace and return, or on their way from their work- 
place to the place where they usually take their meal or attend prayer, and return; (3) any accident incurred by the worker during movements they make for the purpose of doing an assignment required by their employer; and (4) any disease established to be caused by work, as well as any occupational disease diagnosed in accordance with the Schedule of Occupational Diseases (10).

\section{Results}

Data analysis showed the higher percentages of NSIWs in terms of employment $(80.9 \%)$ and suffering of occupational injuries/diseases (93.5\%) as compared to SIWs' employment (19.1\%) and occupational injuries/diseases (6.5\%). Figure 1 shows the overall decreasing trend of occupational injuries/diseases between 2004 and 2013, but the decrease was greater in SIWs. Index value trends of insured workers increased due to their higher share in employment and occupational injuries/diseases.

Index value trends in occupational injuries/diseases in SIWs and NSIWs according to economic activities, based on 2004 as a reference, are shown in Table 1. Trends in occupational injuries/diseases in SIWs and NSIWs decreased for all types of economic activities except financing and real estate. Index value trends in occupational injuries/diseases in NSIWs based on SIWs according to economic activities are shown in Table 2. When we took values of occupational injuries/ diseases in SIWs as a reference, occupational injuries/ diseases among NSIWs increased in construction, followed by trade, financing and real estate, post and communications, and manufacturing, but decreased in agriculture and fishing, and social services.
Index value trends in occupational injuries/diseases in SIWs and NSIWs according to occupation, based on 2004 as a reference, are shown in Table 3. Trends in occupational injuries/diseases in NSIWs increased in engineering, followed by services workers, sales workers and industrial, chemical and food workers. In SIWs, there were increases in industrial, chemical and food workers followed by other occupations and technicians.

Index value trends in occupational injuries/diseases in NSIWs based on SIWs according to major occupations are shown in Table 4. When we took values of occupational injuries/diseases in SIWs as a reference, occupational injuries/diseases among NSIWs increased most for engineering, followed by technicians, administrative and managerial workers, services workers and specialists.

\section{Discussion}

This is believed to be the first national level comparison of occupational injuries/diseases between SIWs and NSIWs. The index value calculation method has already been used in different studies conducted in Turkey, Republic of Korea and Pakistan to determine trends in occupational injuries $(9,11,12)$. Overall, this study found a clear higher percentage share of NSIW with SIW by employment (4 times) and occupational injuries/diseases (14 times). Involvement of NSIWs in more hazardous jobs and higher employment share could explain the higher number of occupational injuries/diseases as higher rates of accidents/ disability have been observed among migrant workers in Qatar and the United Arab Emirates (UAE) (13,14). A higher share of employment and occupational injuries have also been found in other Gulf countries such as Qatar (15) and UAE (16).

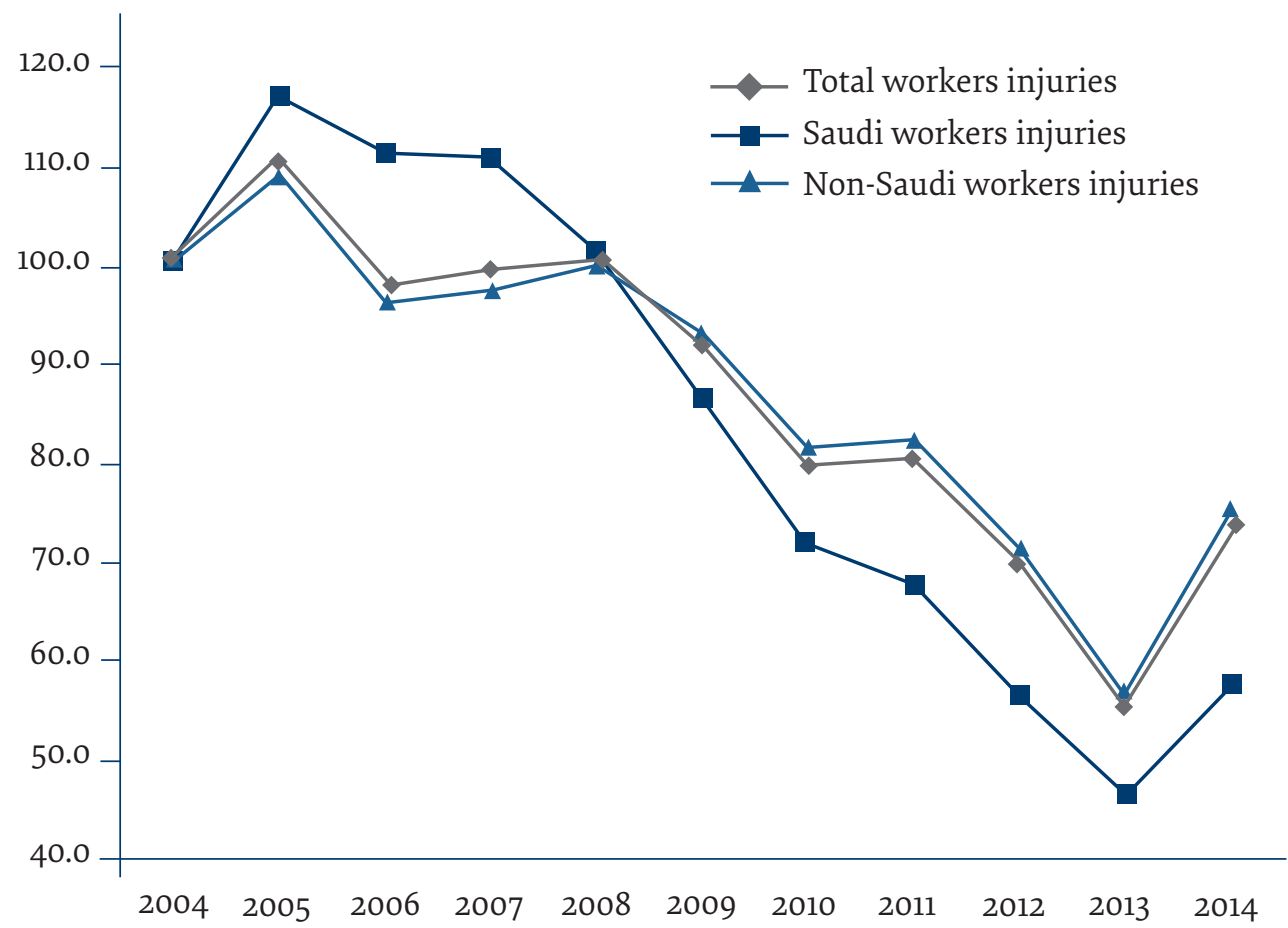




\begin{tabular}{|c|c|c|c|c|c|c|c|c|c|c|c|c|}
\hline Economic activities & 2004 & 2005 & 2006 & 2007 & 2008 & 2009 & 2010 & 2011 & 2012 & 2013 & 2014 & Slope \\
\hline \multicolumn{13}{|l|}{ NSIWs' injuries } \\
\hline \multicolumn{13}{|c|}{ Index value by time, $2004=100$} \\
\hline Post and communications & 100.0 & 111.8 & 97.3 & 110.9 & 90.4 & 78.0 & 66.6 & 67.6 & 54.9 & 50.6 & 76.9 & -5.4 \\
\hline Trade & 100.0 & 114.5 & 104.9 & 101.9 & 84.4 & 68.7 & 65.4 & 80.6 & 72.2 & 42.6 & 53.6 & -6.2 \\
\hline Construction & 100.0 & 107.2 & 94.6 & 98.4 & 113.1 & 110.8 & 96.1 & 93.2 & 79.6 & 68.4 & 91.3 & -2.5 \\
\hline Mining and quarrying & 100.0 & 103.8 & 101.6 & 120.9 & 123.2 & 130.7 & 113.0 & 87.2 & 95.5 & 88.4 & 123.2 & -0.4 \\
\hline Social services & 100.0 & 107.0 & 70.7 & 63.5 & 65.3 & 64.5 & 43.5 & 36.9 & 34.4 & 30.7 & 37.1 & -7.3 \\
\hline Agriculture and fishing & 100.0 & 118.2 & 106.5 & 101.7 & 102.1 & 100.5 & 90.2 & 71.3 & 55.1 & 46.9 & 63.1 & -6.3 \\
\hline Manufacturing & 100.0 & 104.2 & 90.6 & 88.7 & 90.4 & 79.1 & 64.9 & 61.0 & 51.7 & 47.9 & 60.6 & -5.6 \\
\hline Electricity and water & 100.0 & 108.1 & 112.7 & 93.8 & 116.0 & 131.0 & 97.3 & 91.9 & 68.8 & 55.4 & 65.7 & -4.9 \\
\hline Finance and real estate & 100.0 & 117.6 & 153.0 & 301.7 & 369.6 & 472.8 & 611.1 & 489.2 & 461.2 & 388.0 & 713.7 & 51.7 \\
\hline \multicolumn{13}{|l|}{ SIWs' injuries } \\
\hline \multicolumn{13}{|c|}{ Index value by time, $2004=100$} \\
\hline Post and communications & 100.0 & 136.2 & 101.0 & 139.8 & 102.6 & 98.5 & 85.2 & 81.6 & 71.9 & 51.5 & 66.8 & -6.6 \\
\hline Trade & 100.0 & 118.6 & 108.8 & 95.4 & 79.8 & 67.0 & 57.4 & 47.9 & 37.8 & 30.3 & 35.0 & -9.2 \\
\hline Construction & 100.0 & 127.9 & 121.8 & 126.7 & 110.4 & 84.2 & 70.3 & 64.1 & 53.8 & 47.0 & 55.4 & -8.3 \\
\hline Mining and quarrying & 100.0 & 131.0 & 126.4 & 134.4 & 144.7 & 120.2 & 90.2 & 99.0 & 72.9 & 76.5 & 128.2 & -3.3 \\
\hline Social services & 100.0 & 134.7 & 131.6 & 129.3 & $115 \cdot 5$ & 101.3 & 98.3 & 104.7 & 101.3 & 94.6 & 125.6 & -1.7 \\
\hline Agriculture and fishing & 100.0 & 96.7 & 101.7 & 126.7 & 125.0 & 111.7 & 105.0 & 113.3 & 138.3 & 105.0 & 101.7 & 1.0 \\
\hline Manufacturing & 100.0 & 110.6 & 106.8 & 106.1 & 101.7 & 86.2 & 72.0 & 66.8 & 56.3 & 43.9 & 49.1 & -7.1 \\
\hline Electricity and water & 100.0 & $95 \cdot 4$ & 92.6 & 88.1 & 86.4 & 91.2 & 59.2 & 54.2 & 46.3 & $33 \cdot 3$ & 46.7 & -6.8 \\
\hline Finance and real estate & 100.0 & 114.7 & 185.3 & 379.4 & 376.5 & 305.9 & 367.6 & 438.2 & 352.9 & 276.5 & 441.2 & 27.0 \\
\hline
\end{tabular}

NSIWs = non-Saudi insured workers; SIWs = Saudi insured workers.

The greater decrease in occupational injuries/diseases among SIWs as compared to NSIWs is consistent with another study (17). The downward trend in index values of occupational injuries could also have been due to the steps taken by GOSI such as: awarding occupational health and safety (OHS) prizes to establishments, providing technical and regulatory assistance, training programmes, OHS workshops, field studies about OHS and strict inspection of premises.

The outcomes of this study illustrate that, as compared to SIWs, the increased index value trends in occupational injuries/diseases of NSIWs show the related suffering from work conditions or unsafe practices. The global construction sector is the most hazardous for occupational accidents/injuries, with extensive involvement of migrant workers. It has been shown that migrant workers in the construction sector have a significantly high proportion (42\%) of severe occupational injuries, which is consistent with our study (18). After construction, trade and manufacturing economic activities were the second and third most problematic groups, respectively, regarding incidence of occupational injuries/diseases among NSIWs. This could be associated with the growing industrial activities in Saudi Arabia.

Between 2004 and 2014 occupational injuries/diseases increased in mining and quarrying in SIWs and NSIWs, which could be associated with the hazardous nature of oil exploration activities. Increased trends in occupational injuries/diseases in NSIWs in trade, financing and real estate, manufacturing, and agriculture and fishing may have been due to the high level of NSIW involvement in blue collar jobs and lack of interest in these jobs from SIWs.

The distribution of occupational injuries/diseases among migrant workers varied with the nature of the occupation. The most hazardous occupations regarding injuries/diseases were engineering and services among SIWs and NSIWs. The increased trend in occupational injuries for SIWs and NSIWs among industrial, chemical and food workers could have been due to inadequate preventive measures. A recent study reported that occupational injuries among migrant workers were due to stress and depression, which may have been due to job choice and being away from their home country (18). Trends in occupational injuries/diseases increased in Saudi and non-Saudi technicians.

Our study had some limitations regarding types of occupational injuries, age of the working population, and gender and nationality distribution of the migrant workers. A major strength of our study was that the findings are of national importance concerning occupational injury/disease burden among SIWs and NSIWs. 


\begin{tabular}{|c|c|c|c|c|c|c|c|c|c|c|c|c|c|}
\hline \multicolumn{2}{|l|}{$\begin{array}{l}\text { Economic } \\
\text { activities }\end{array}$} & 2004 & 2005 & 2006 & 2007 & 2008 & 2009 & 2010 & 2011 & 2012 & 2013 & 2014 & Slope \\
\hline \multicolumn{14}{|c|}{ Index value by nationality, occupational injuries/diseases value of SIWs = 100} \\
\hline \multirow{3}{*}{$\begin{array}{l}\text { Post and } \\
\text { communications }\end{array}$} & SIWs & 196.0 & 267.0 & 198.0 & 274.0 & 201.0 & 193.0 & 167.0 & 160.0 & 141.0 & 101.0 & 131.0 & \\
\hline & NSIWs & 2006.0 & 2243.0 & 1952.0 & 2225.0 & 1813.0 & 1564.0 & 1335.0 & 1357.0 & 1102.0 & 1016.0 & 1542.0 & \\
\hline & Index value & 1023.5 & 840.1 & 985.9 & 812.0 & 902.0 & 810.4 & 799.4 & 848.1 & 781.6 & 1005.9 & 1177.1 & 7.2 \\
\hline \multirow[t]{3}{*}{ Trade } & SIWs & 1555.0 & 1845.0 & 1692.0 & 1483.0 & 1241.0 & 1042.0 & 893.0 & 745.0 & 588.0 & 471.0 & 544.0 & \\
\hline & NSIWs & 23125.0 & 26470.0 & 24254.0 & 23559.0 & 19525.0 & 15897.0 & 15135.0 & 18640.0 & 16687.0 & 9841.0 & 12404.0 & \\
\hline & Index value & 1487.1 & 1434.7 & 1433.5 & 1588.6 & 1573.3 & 1525.6 & 1694.8 & 2502.0 & 2837.9 & 2089.4 & 2280.1 & 115.9 \\
\hline \multirow[t]{3}{*}{ Construction } & SIWs & 868.0 & 1110.0 & 1057.0 & 1100.0 & 958.0 & 731.0 & 610.0 & 556.0 & 467.0 & 408.0 & 481.0 & \\
\hline & NSIWs & 38431.0 & 41216.0 & 36370.0 & 37829.0 & 43472.0 & 42577.0 & 36917.0 & 35811.0 & 30581.0 & 26292.0 & 35071.0 & \\
\hline & Index value & 4427.5 & 3713.2 & 3440.9 & 3439.0 & 4537.8 & 5824.5 & 6052.0 & 6440.8 & 6548.4 & 6444.1 & 7291.3 & 382.6 \\
\hline \multirow{3}{*}{$\begin{array}{l}\text { Mining and } \\
\text { quarrying }\end{array}$} & SIWs & 387.0 & 507.0 & 489.0 & 520.0 & 560.0 & 465.0 & 349.0 & 383.0 & 282.0 & 296.0 & 496.0 & \\
\hline & NSIWs & 690.0 & 716.0 & 701.0 & 834.0 & 850.0 & 902.0 & 780.0 & 602.0 & 659.0 & 610.0 & 850.0 & \\
\hline & Index value & 178.3 & 141.2 & 143.4 & 160.4 & 151.8 & 194.0 & 223.5 & 157.2 & 233.7 & 206.1 & 171.4 & 5.1 \\
\hline \multirow[t]{3}{*}{ Social services } & SIWs & 297.0 & 400.0 & 391.0 & 384.0 & 343.0 & 301.0 & 292.0 & 311.0 & 301.0 & 281.0 & 373.0 & \\
\hline & NSIWs & 4005.0 & 4286.0 & 2832.0 & 2543.0 & 2617.0 & 2584.0 & 1741.0 & 1478.0 & 1376.0 & 1230.0 & 1487.0 & \\
\hline & Index value & 1348.5 & 1071.5 & 724.3 & 662.2 & 763.0 & 858.5 & 596.2 & 475.2 & 457.1 & 437.7 & 398.7 & -78.4 \\
\hline \multirow{3}{*}{$\begin{array}{l}\text { Agriculture and } \\
\text { fishing }\end{array}$} & SIWs & 60.0 & 58.0 & 61.0 & 76.0 & 75.0 & 67.0 & 63.0 & 68.0 & 83.0 & 63.0 & 61.0 & \\
\hline & NSIWs & 757.0 & 895.0 & 806.0 & 770.0 & 773.0 & 761.0 & 683.0 & 540.0 & 417.0 & 355.0 & 478.0 & \\
\hline & Index value & 1261.7 & 1543.1 & 1321.3 & 1013.2 & 1030.7 & 1135.8 & 1084.1 & 794.1 & 502.4 & 563.5 & 783.6 & -83.2 \\
\hline \multirow[t]{3}{*}{ Manufacturing } & SIWs & 2529.0 & 2798.0 & 2701.0 & 2684.0 & 2572.0 & 2180.0 & 1820.0 & 1690.0 & 1425.0 & 1110.0 & 1242.0 & \\
\hline & NSIWs & 16774.0 & 17485.0 & 15191.0 & 14886.0 & 15169.0 & 13274.0 & 10894.0 & 10231.0 & 8678.0 & 8038.0 & 10158.0 & \\
\hline & Index value & 663.3 & 624.9 & 562.4 & 554.6 & 589.8 & 608.9 & 598.6 & 605.4 & 609.0 & 724.1 & 817.9 & 12.9 \\
\hline \multirow{3}{*}{$\begin{array}{l}\text { Electricity and } \\
\text { water }\end{array}$} & SIWs & 544.0 & 519.0 & 504.0 & 479.0 & 470.0 & 496.0 & 322.0 & 295.0 & 252.0 & 181.0 & 254.0 & \\
\hline & NSIWs & 848.0 & 917.0 & 956.0 & 795.0 & 984.0 & 1111.0 & 825.0 & 779.0 & 583.0 & 470.0 & 557.0 & \\
\hline & Index value & 155.9 & 176.7 & 189.7 & 166.0 & 209.4 & 224.0 & 256.2 & 264.1 & 231.3 & 259.7 & 219.3 & 9.2 \\
\hline \multirow{3}{*}{$\begin{array}{l}\text { Finance and real } \\
\text { estate }\end{array}$} & SIWs & 34.0 & 39.0 & 63.0 & 129.0 & 128.0 & 104.0 & 125.0 & 149.0 & 120.0 & 94.0 & 150.0 & \\
\hline & NSIWs & 415.0 & 488.0 & 635.0 & 1252.0 & 1534.0 & 1962.0 & 2536.0 & 2030.0 & 1914.0 & 1610.0 & 2962.0 & \\
\hline & Index value & 1220.6 & 1251.3 & 1007.9 & 970.5 & 1198.4 & 1886.5 & 2028.8 & 1362.4 & 1595.0 & 1712.8 & 1974.7 & 81.7 \\
\hline
\end{tabular}

NSIWs = non-Saudi insured workers; SIWs = Saudi insured workers.

\section{Conclusions}

We found a high level of occupational injuries/diseases among NSIWs, which needs to be addressed through an effective injury prevention programme, especially for the construction sector, and engineering and services workers. The decline in overall occupational injuries/diseases burden could be associated with positive steps taken by GOSI. The burden of occupational injuries/diseases among NSIWs could be reduced over time through government and private sector partnerships, occupational safety education, and minimizing language barriers. Future studies could be designed to investigate the severity of occupational injuries/diseases among SIWs and NSIWs.

Funding: None.

Competing interests: None declared. 


\begin{tabular}{|c|c|c|c|c|c|c|c|c|c|c|c|}
\hline Major occupations & 2004 & 2005 & 2006 & 2007 & 2008 & 2009 & 2010 & 2011 & 2013 & 2014 & Slope \\
\hline \multicolumn{12}{|l|}{ NSIWs' injuries } \\
\hline \multicolumn{12}{|l|}{ Index value by time, $2004=100$} \\
\hline Administrative and managerial & 100.0 & 6.0 & 5.1 & 7.1 & 12.0 & 18.6 & 21.7 & 19.9 & 21.1 & 21.8 & -2.9 \\
\hline Specialists & 100.0 & 181.5 & $157 \cdot 3$ & 157.8 & $157 \cdot 3$ & 146.2 & 133.8 & 126.9 & 99.9 & 130.4 & -3.2 \\
\hline Technicians & 100.0 & 105.9 & 115.6 & 162.0 & 238.5 & 278.6 & 290.2 & 339.9 & 260.6 & 335.6 & 28.8 \\
\hline Clerical and related & 100.0 & 4.6 & 3.7 & 3.2 & 2.7 & 2.0 & 1.7 & 1.3 & 0.9 & 1.0 & -5.7 \\
\hline Sales & 100.0 & 1357.4 & 1127.8 & 1088.9 & 972.2 & 864.8 & 674.1 & 609.3 & 557.4 & 844.4 & -17.2 \\
\hline Services & 100.0 & 8448.0 & 7127.4 & 6880.0 & 5898.1 & 4742.2 & 3773.2 & 3523.2 & 2413.7 & 3613.1 & -237.1 \\
\hline Agriculture & 100.0 & 0.6 & 0.5 & 0.5 & 0.6 & 0.6 & 0.5 & 0.5 & 0.4 & 0.7 & -5.4 \\
\hline Industrial, chemical and food & 100.0 & 343.5 & 341.5 & 414.9 & 599.2 & 726.6 & 689.5 & 721.0 & 654.4 & 685.5 & 62.4 \\
\hline Engineering & 100.0 & 5214.5 & 4941.4 & 5269.4 & 6459.6 & 6635.3 & 6141.5 & 6428.5 & 4309.3 & 5343.0 & 309.6 \\
\hline Other occupations & 100.0 & 65.7 & 61.2 & 62.4 & 23.0 & 14.6 & 9.6 & 18.0 & 39.3 & 66.3 & $-5 \cdot 3$ \\
\hline \multicolumn{12}{|l|}{ SIWs' injuries } \\
\hline \multicolumn{12}{|l|}{ Index value by time, $2004=100$} \\
\hline Administrative and managerial & 100.0 & 83.7 & 67.3 & 85.7 & 136.7 & 202.0 & 144.9 & 163.3 & 134.7 & 140.8 & 8.8 \\
\hline Specialists & 100.0 & 96.6 & 88.1 & 91.5 & 93.2 & 86.4 & 61.0 & 63.6 & 53.4 & 86.4 & -3.9 \\
\hline Technicians & 100.0 & 270.7 & $345 \cdot 5$ & 479.8 & 521.2 & 491.9 & 487.9 & 514.1 & 348.5 & 448.5 & 27.4 \\
\hline Clerical and related & 100.0 & 105.2 & 83.7 & 81.5 & $65 \cdot 3$ & 53.8 & 45.2 & 38.7 & 31.8 & 45.8 & -8.2 \\
\hline Sales & 100.0 & 134.4 & 100.8 & 133.6 & 141.8 & 108.2 & 86.1 & 75.4 & 59.8 & 72.1 & -6.5 \\
\hline Services & 100.0 & 113.5 & 107.8 & 103.5 & 87.5 & 69.9 & 54.3 & 49.1 & 31.4 & 37.9 & -9.6 \\
\hline Agriculture & 100.0 & 46.2 & 84.6 & 69.2 & 61.5 & 107.7 & 100.0 & 138.5 & 130.8 & 184.6 & 10.7 \\
\hline Industrial, chemical and food & 100.0 & 269.1 & 238.2 & 309.1 & 356.4 & 518.2 & 450.9 & 525.5 & 478.2 & $525 \cdot 5$ & $44 \cdot 3$ \\
\hline Engineering & 100.0 & 116.3 & 114.5 & 106.1 & 104.8 & 86.1 & 76.9 & 69.1 & 44.7 & 50.6 & -7.8 \\
\hline Other occupations & 100.0 & 106.3 & 165.6 & 362.5 & 387.5 & 390.6 & 225.0 & 262.5 & 168.8 & 459.4 & 22.7 \\
\hline
\end{tabular}

NSIWs = non-Saudi insured workers; SIWs = Saudi insured workers. 


\begin{tabular}{|c|c|c|c|c|c|c|c|c|c|c|c|}
\hline $\begin{array}{l}\text { Major occupations } \\
\text { (Index value } \\
\text { by nationality, } \\
\text { occupational } \\
\text { injuries/diseases } \\
\text { value of SIWs = 100) }\end{array}$ & 2004 & 2005 & 2006 & 2007 & 2008 & 2009 & 2010 & 2011 & 2013 & 2014 & Slope \\
\hline \multicolumn{12}{|c|}{ Administrative and managerial } \\
\hline SIWs & 49.0 & 41.0 & 33.0 & 42.0 & 67.0 & 99.0 & 71.0 & 80.0 & 66.0 & 69.0 & \\
\hline NSIWs & 54.0 & 70.0 & 59.0 & 83.0 & 140.0 & 217.0 & 253.0 & 232.0 & 246.0 & 254.0 & \\
\hline Index value & 110.2 & 170.7 & 178.8 & 197.6 & 209.0 & 219.2 & 356.3 & 290.0 & 372.7 & 368.1 & 29.0 \\
\hline \multicolumn{12}{|l|}{ Specialists } \\
\hline SIWs & 118.0 & 114.0 & 104.0 & 108.0 & 110.0 & 102.0 & 72.0 & 75.0 & 63.0 & 102.0 & \\
\hline NSIWs & 1164.0 & 1229.0 & 1065.0 & 1068.0 & 1065.0 & 990.0 & 906.0 & 859.0 & 676.0 & 883.0 & \\
\hline Index value & 986.4 & 1078.1 & 1024.0 & 988.9 & 968.2 & 970.6 & 1258.3 & 1145.3 & 1073.0 & 865.7 & 1.8 \\
\hline \multicolumn{12}{|l|}{ Technicians } \\
\hline SIWs & 99.0 & 268.0 & 342.0 & 475.0 & 516.0 & 487.0 & 483.0 & 509.0 & 345.0 & 444.0 & \\
\hline NSIWs & 677.0 & 1385.0 & 1512.0 & 2119.0 & 3120.0 & 3644.0 & 3796.0 & 4446.0 & 3408.0 & 4389.0 & \\
\hline Index value & 683.8 & 516.8 & 442.1 & 446.1 & 604.7 & 748.3 & 785.9 & 873.5 & 987.8 & 988.5 & 56.7 \\
\hline \multicolumn{12}{|l|}{ Clerical and related } \\
\hline SIWs & 877.0 & 923.0 & 734.0 & 715.0 & 573.0 & 472.0 & 396.0 & 339.0 & 279.0 & 402.0 & \\
\hline NSIWs & 1308.0 & 1311.0 & 1053.0 & 920.0 & 788.0 & 589.0 & 481.0 & 382.0 & 256.0 & 284.0 & \\
\hline Index value & 149.1 & 142.0 & 143.5 & 128.7 & 137.5 & 124.8 & 121.5 & 112.7 & 91.8 & 70.6 & -7.6 \\
\hline \multicolumn{12}{|l|}{ Sales } \\
\hline SIWs & 122.0 & 164.0 & 123.0 & 163.0 & 173.0 & 132.0 & 105.0 & 92.0 & 73.0 & 88.0 & \\
\hline NSIWs & 671.0 & 733.0 & 609.0 & 588.0 & 525.0 & 467.0 & 364.0 & 329.0 & 301.0 & 456.0 & \\
\hline Index value & 550.0 & 447.0 & 495.1 & 360.7 & 303.5 & 353.8 & 346.7 & 357.6 & 412.3 & 518.2 & -7.3 \\
\hline \multicolumn{12}{|l|}{ Services } \\
\hline SIWs & 3288.0 & 3731.0 & 3544.0 & 3404.0 & 2877.0 & 2298.0 & 1784.0 & 1616.0 & 1033.0 & 1247.0 & \\
\hline NSIWs & 53339.0 & 56686.0 & 47825.0 & 46165.0 & 39576.0 & 31820.0 & 25318.0 & 23641.0 & 16196.0 & 24244.0 & \\
\hline Index value & 1622.2 & 1519.3 & 1349.5 & 1356.2 & 1375.6 & 1384.7 & 1419.2 & 1462.9 & 1567.9 & 1944.2 & 24.3 \\
\hline \multicolumn{12}{|l|}{ Agriculture } \\
\hline SIWs & 13.0 & 6.0 & 11.0 & 9.0 & 8.0 & 14.0 & 13.0 & 18.0 & 17.0 & 24.0 & \\
\hline NSIWs & 248.0 & 316.0 & 278.0 & 256.0 & 334.0 & 336.0 & 292.0 & 288.0 & 227.0 & 375.0 & \\
\hline Index value & 1907.7 & 5266.7 & 2527.3 & 2844.4 & 4175.0 & 2400.0 & 2246.2 & 1600.0 & 1335.3 & 1562.5 & \\
\hline \multicolumn{12}{|c|}{ Industrial, chemical and food } \\
\hline SIWs & 55.0 & 148.0 & 131.0 & 170.0 & 196.0 & 285.0 & 248.0 & 289.0 & 263.0 & 289.0 & \\
\hline NSIWs & 614.0 & 852.0 & 847.0 & 1029.0 & 1486.0 & 1802.0 & 1710.0 & 1788.0 & 1623.0 & 1700.0 & \\
\hline Index value & 1116.4 & 575.7 & 646.6 & 605.3 & 758.2 & 632.3 & 689.5 & 618.7 & 617.1 & 588.2 & -27.1 \\
\hline \multicolumn{12}{|l|}{ Engineering } \\
\hline SIWs & 1817.0 & 2114.0 & 2081.0 & 1927.0 & 1904.0 & 1565.0 & 1397.0 & 1255.0 & 812.0 & 920.0 & \\
\hline NSIWs & 28798.0 & 32017.0 & 30340.0 & 32354.0 & 39662.0 & 40741.0 & 37709.0 & 39471.0 & 26459.0 & 32806.0 & \\
\hline Index value & 1584.9 & 1514.5 & 1458.0 & 1679.0 & 2083.1 & 2603.3 & 2699.3 & 3145.1 & 3258.5 & 3565.9 & 254.9 \\
\hline \multicolumn{12}{|l|}{ Other occupations } \\
\hline SIWs & 32.0 & 34.0 & 53.0 & 116.0 & 124.0 & 125.0 & 72.0 & 84.0 & 54.0 & 147.0 & \\
\hline NSIWs & 178.0 & 117.0 & 109.0 & 111.0 & 41.0 & 26.0 & 17.0 & 32.0 & 70.0 & 118.0 & \\
\hline Index value & 556.3 & 344.1 & 205.7 & 95.7 & 33.1 & 20.8 & 23.6 & 38.1 & 129.6 & 80.3 & -41.5 \\
\hline
\end{tabular}




\section{Tendances des accidents du travail et des maladies professionnelles chez les travailleurs assurés saoudiens et non saoudiens}

\section{Résumé}

Contexte: Le développement actuel des industries et des infrastructures en Arabie saoudite est associé à un risque élevé d'accidents du travail et de maladies professionnelles.

Objectif : La présente étude avait pour objectif de comparer les tendances des accidents du travail et des maladies professionnelles chez les travailleurs assurés saoudiens et non saoudiens.

Méthodes : Nous avons utilisé la méthode des valeurs d'indice et le calcul de la pente pour analyser les tendances des accidents du travail et des maladies professionnelles à partir des données des rapports statistiques annuels publiés par l'Organisation générale de l'assurance sociale entre 2004 et 2014 . Nous avons analysé les données portant sur 10565993 travailleurs assurés saoudiens (18,5\%) et 46402079 travailleurs assurés non saoudiens (81,5\%) ainsi que sur 896627 accidents du travail et maladies professionnelles.

Résultats : La distribution des incidences des accidents du travail et des maladies professionnelles chez les travailleurs assurés non saoudiens (93,5\%) était 14 fois plus importante que chez les travailleurs assurés saoudiens (6,5\%). Les accidents du travail et les maladies professionnelles étaient plus susceptibles d'augmenter chez les travailleurs assurés non saoudiens que chez les travailleurs assurés saoudiens, en particulier dans les secteurs économiques de la construction, du commerce, de la finance et de l'immobilier, dans les professions d'ingénieur et de technicien et dans le secteur des services. Jusqu'en 2013, on observait une tendance globale à la baisse des accidents du travail et des maladies professionnelles.

Conclusions : La mise en place d'un programme de prévention efficace s'impose donc pour réduire le risque élevé d'accidents du travail et de maladies professionnelles auquel sont exposés les travailleurs assurés non saoudiens.

$$
\begin{aligned}
& \text { الإصابات والأمر اض المهنية بين السعوديين وغير السعوديين العاملين المؤمن عليهم } \\
& \text { محسن عباس، محمد كاشف، منصور بالخيور، إعجاز أحمد، زكي الزمان عصام، راشد سعيد } \\
& \text { الخالاصة } \\
& \text { الخلفية: إن التنمية الصناعية والبنية التحتية المتو اصلة في المملكة العربية السعودية تحمل خخاطر عالية للإصابات والأمراض المهنية. } \\
& \text { الهدف: هدفت الدراسة إلى مقارنة اتجاهات الإصابات والأمراض المهنية بين السعوديين وغير السعوديين العاملين المؤمن عليهم }
\end{aligned}
$$

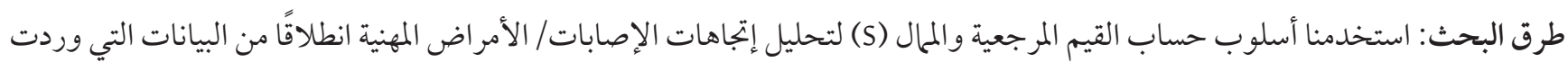

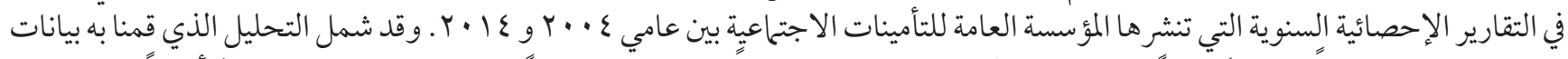

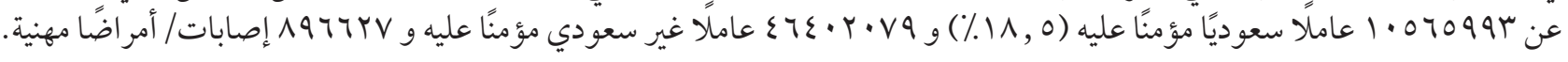

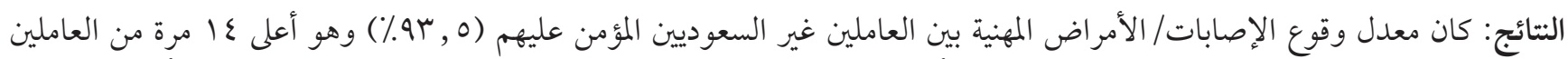

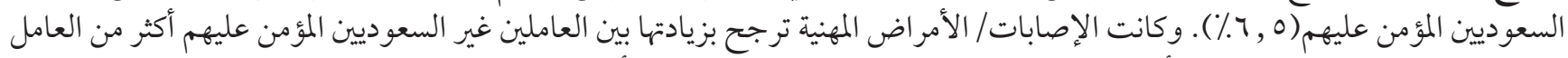

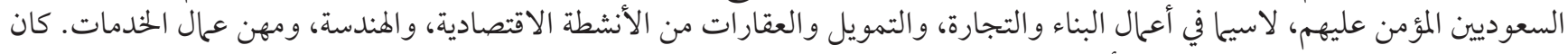

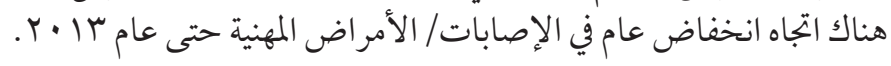

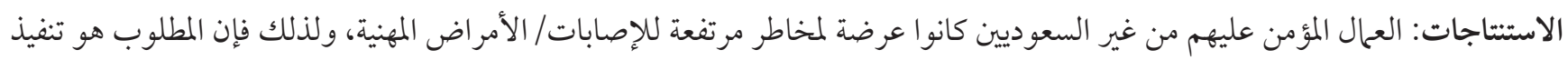

$$
\begin{aligned}
& \text { برنامج للوقاية الفعالة من الإصابة. }
\end{aligned}
$$

\section{References}

1. Salvatore MA, Baglio G, Cacciani L, Spagnolo A, Rosano A. Work-related injuries among immigrant workers in Italy. J Immigr Minor Health. 2013 Feb;15(1):182-7. https://doi.org/10.1007/s10903-012-9673-8 PMID:22752688

2. Marín AJ, Grzywacz JG, Arcury TA, Carrillo L, Coates ML, Quandt SA. Evidence of organizational injustice in poultry processing plants: Possible effects on occupational health and safety among Latino workers in North Carolina. Am J Ind Med. 2009 Jan;52(1):37-48. https://doi.org/10.1002/ajim.20643 PMID:18942666

3. Dong X, Ringen K, Men Y, Fujimoto A. Medical costs and sources of payment for work-related injuries among Hispanic construction workers. J Occup Environ Med. 2007 Dec;49(12):1367-75. https://doi.org/10.1097/JOM.obo13e31815796a8 PMID:18231083

4. Grzywacz JG, Arcury TA, Marín A, Carrillo L, Coates ML, Burke B, et al. The organization of work: implications for injury and illness among immigrant Latino poultry-processing workers. Arch Environ Occup Health. 2007 Spring;62(1):19-26. https://doi. org/10.3200/AEOH.62.1.19-26 PMID:18171643

5. Ahonen EQ, Benavides FG. Risk of fatal and non-fatal occupational injury in foreign workers in Spain. J Epidemiol Community Health. 2006 May;60(5):424-6. https://doi.org/10.1136/jech.2005.044099 PMID:16614333 
6. Salminen S. Are immigrants at increased risk of occupational injury? A literature review. Ergon Open J. 2011;4(1):125-30. https:// doi.org/10.2174/1875934301104010125

7. López-Jacob MJ, Safont EC, García AM, Garí A, Agudelo-Suárez A, Gil A, et al. Participation and influence of migrant workers on working conditions: a qualitative approach. New Solut. 2010;20(2):225-38. https://doi.org/10.2190/NS.20.2.g PMID:20621886

8. General Authority for Statistics [website] (https://www.stats.gov.sa/sites/default/files/mwshrt_lqw_lml_1999-2016-lmwq_llktrwny.pdf, accessed 19 June 2018).

9. Abbas M. Trend of occupational injuries/diseases in Pakistan: index value analysis of injured employed persons from $2001-02$ to 2012-13. Saf Health Work. 2015 Sep;6(3):218-26. https://doi.org/10.1016/j.shaw.2015.05.004 PMID:26929831

10. Insurance terms [website]. Riyadh: General Organization for Social Insurance; 2017 (http://www.gosi.gov.sa/portal/web/guest/ policy/terminology,date accessed 29 May 2018)

11. Unsar S, Sut N. General assessment of the occupational accidents that occurred in Turkey between the years 2000 and 2005 . Saf Sci 2009; 47:614e9. https://doi.org/10.1016/j.ssci.2008.08.001.

12. Rhee KY, Choe SW, Kim YS, Koo KH. The trend of occupational injuries in Korea from 2001 to 2010. Saf Health Work 2013; 4:63e70. https://doi.org/10.5491/SHAW.2013.4.1.63.

13. Byler CG. Hispanic/Latino fatal occupational injury rates. Mon Labor Rev. 2013;136:14-23.

14. Premji S, Messing K, Lippel K. Broken English, broken bones? Mechanisms linking language proficiency and occupational health in a Montreal garment factory. Int J Health Serv. 2008;38(1):1-19. https://doi.org/10.2190/HS.38.1.a PMID:18341120

15. Al-Thani H, El-Menyar A, Consunji R, Mekkodathil A, Peralta R, Allen KA, et al. Epidemiology of occupational injuries by nationality in Qatar: evidence for focused occupational safety programmes. Injury. 2015 Sep;46(9):1806-13. https://doi.org/10.1016/j. injury.2015.04.023 PMID:25943291

16. Barss P, Addley K, Grivna M, Stanculescu C, Abu-Zidan F. Occupational injury in the United Arab Emirates: epidemiology and prevention. Occup Med (Lond). 2009 Oct;59(7):493-8. https://doi.org/10.1093/occmed/kqp101 PMID:19640929

17. Hämäläinen P, Leena Saarela K, Takala J. Global trend according to estimated number of occupational accidents and fatal work-related diseases at region and country level. J Safety Res. 2009;40(2):125-39. https://doi.org/10.1016/j.jsr.2008.12.010 PMID:19433205

18. Al-Thani H, El-Menyar A, Abdelrahman H, Zarour A, Consunji R, Peralta R, et al. Workplace-related traumatic injuries: insights from a rapidly developing Middle Eastern country. J Environ Public Health. 2014;2014:430832. https://doi.org/10.1155/2014/430832 PMID:24734049 